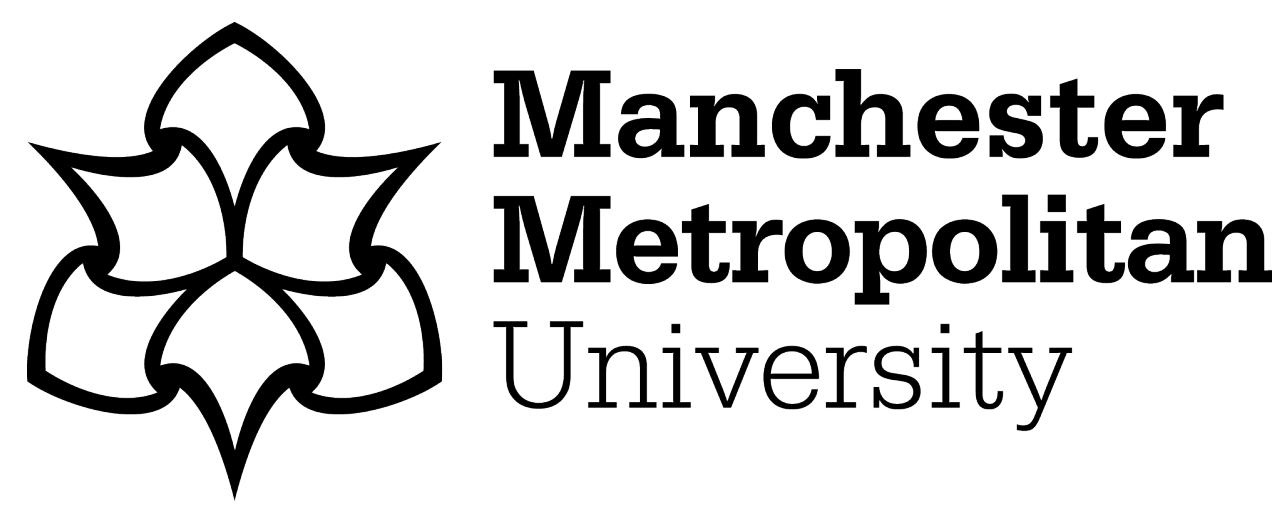

Sheen, KA ORCID logoORCID: https://orcid.org/0000-0002-6326-3241, Luximon, Y, Fung, KH, Chak, SH, Chiu, WY and Chan, WS (2018) Usability Study and Redesign of the Food Tray. In: Advances in Usability, User Experience and Assistive Technology. International Conference on Applied Human Factors and Ergonomics (AHFE 2018), 21 July 2018 - 25 July 2018, Orlando, USA.

Downloaded from: https://e-space.mmu.ac.uk/625467/

Publisher: Springer

DOI: https://doi.org/10.1007/978-3-319-94947-5_40

Please cite the published version 


\title{
Usability Study and Redesign of the Food Tray
}

\author{
Kimberly Anne Sheen ${ }^{1, *}$, Yan Luximon ${ }^{1}$, Kar Hei Fung ${ }^{1}$, Shun Him Chak ${ }^{1}$, \\ Wai Yi Chiu ${ }^{1}$ and Wing Sang Chan ${ }^{1}$ \\ ${ }^{1}$ School of Design, The Hong Kong Polytechnic University \\ Hung Hom, Kowloon, Hong Kong SAR \\ \{kaasheen, yan.luximon\}@ polyu.edu.hk \\ \{15082378D, 17040384D, 17035441D, 17040499D\}@connect.polyu.hk
}

\begin{abstract}
Food trays can be found around the world in schools, canteens, and restaurants. While useful for carrying food to a table, the design has caused frustration in countless users. The aim of the research outlined in this paper was to identify design criteria for food trays and produce a redesign to improve the user experience with an emphasis on ergonomics. The investigation focused on four areas: loading, carrying, eating from, and cleaning and storing the food tray. Areas of concern and design criteria were found through materials research, observations, questionnaires, interviews, and prototype testing. Details of the findings and the ergonomically designed prototype are presented in this paper. By investigating food trays from the user and staff perspective, design criteria and a unique design were developed which users felt was more comfortable and fit cleaning and storage requirements.
\end{abstract}

Keywords: Usability study $\cdot$ Observations $\cdot$ Interviews $\cdot$ Future design

\section{$1 \quad$ Introduction}

Food trays are a common staple in schools, mall canteens, and restaurants. While used around the world, there tends to be very little variation in their design. While useful for carrying food to a table, the design has caused frustration to countless users while not only carrying the food but through all steps of use, yet there is very little research into this.

Research surrounding food trays tends to be very specific, such as prototyping and creating food trays to facilitate self-feeding in young children [1]. Even in the news, discussions surround food trays tend to be related to specialty trays such as airplanes and tied to their economic value [2]. The most recent news surrounding this common product was related to a trend in Singapore where they are starting to charge a deposit

\footnotetext{
* Please note that the LNCS Editorial assumes that all authors have used the western naming convention, with given names preceding surnames (first name then last name). This determines the structure of the names in the running heads and the author index. No academic titles or descriptions of academic positions should be included in the addresses. The affiliations should consist of the author's institution, town, and country.
} 
fee for the use of the trays. This has been so unpopular with the consumers, that $50 \%$ to $90 \%$ of patrons asked to pay the fee refused to take trays and instead moved their food in trips [3] or returned the trays yet left the plates and cutlery on the tables [4].

While trays are meant to ease the movement of food from a counter to a table, it is telling when people are willing to go without them when they feel they are inconvenienced with a returnable fee of 50 cents. And even though there have been patents for unique designs of food trays $[5,6,7]$, they have not been adopted as the standard food tray. Taking all of this into account, the goal of the research project outlined in this paper was to identify specific ergonomic issues related to the design of food trays, identify other areas of concern in the design of food trays, and produce a redesign to improve the user experience with an emphasis on ergonomics. This was done by ensuring the new design was fit for usage throughout the 4 main stages of use: loading food, carrying the tray, eating, and after-use management (to collect, clean, and store the tray).

\section{Redesign Process}

First, the identification of areas of concern and the criteria for the redesign were identified through several methods. These methods are as follows: materials research, observations, questionnaire, and interviews. Following this, usability testing with prototypes was conducted in two rounds. The investigation focused on four main areas: loading of the food tray, carrying a full tray of food, eating from the food tray, and the afteruse management which includes cleaning and storage.

The investigation took place at one of the cafeterias located on a university campus. This study not only looked at the users of the food tray throughout their use experience but also at the staff who are tasked with cleaning and storing the food trays when they are not in use. Investigation began with observations of staff and users and materials research, which was then followed by an in-depth questionnaire. From this information, initial design criteria were developed. Following that, a prototype was developed and tested with users. Interviews were conducted during these user tests and data gathered was used to iterate the prototypes and create a final design. This final design and the criteria are presented in this paper.

\subsection{Development of Design Criteria}

Research into problem identification started with a review of existing products. This included the existing common food tray, patents for unique food tray designs, materials, and features in similar products. This research not only identified design criteria, but also looked into limitations applicable to the design. Following which, general observations of 13 people using the canteen was undertaken. Photos and notes were taken to identify the steps associated with using the food trays and issues that users encountered. Additionally, an in-depth online questionnaire was released to students and staff regarding usage of food trays. 41 respondents outlined their experience with food trays at the university. As diners are not the only stakeholders of the food tray, design criteria research was undertaken with canteen staff as well. Four members of canteen staff were interviewed about their experiences with the food trays and the cleaning and storage 
process. Additionally, researchers were allowed to observe the cleaning and storage process which the food trays go through (see Figure 1).

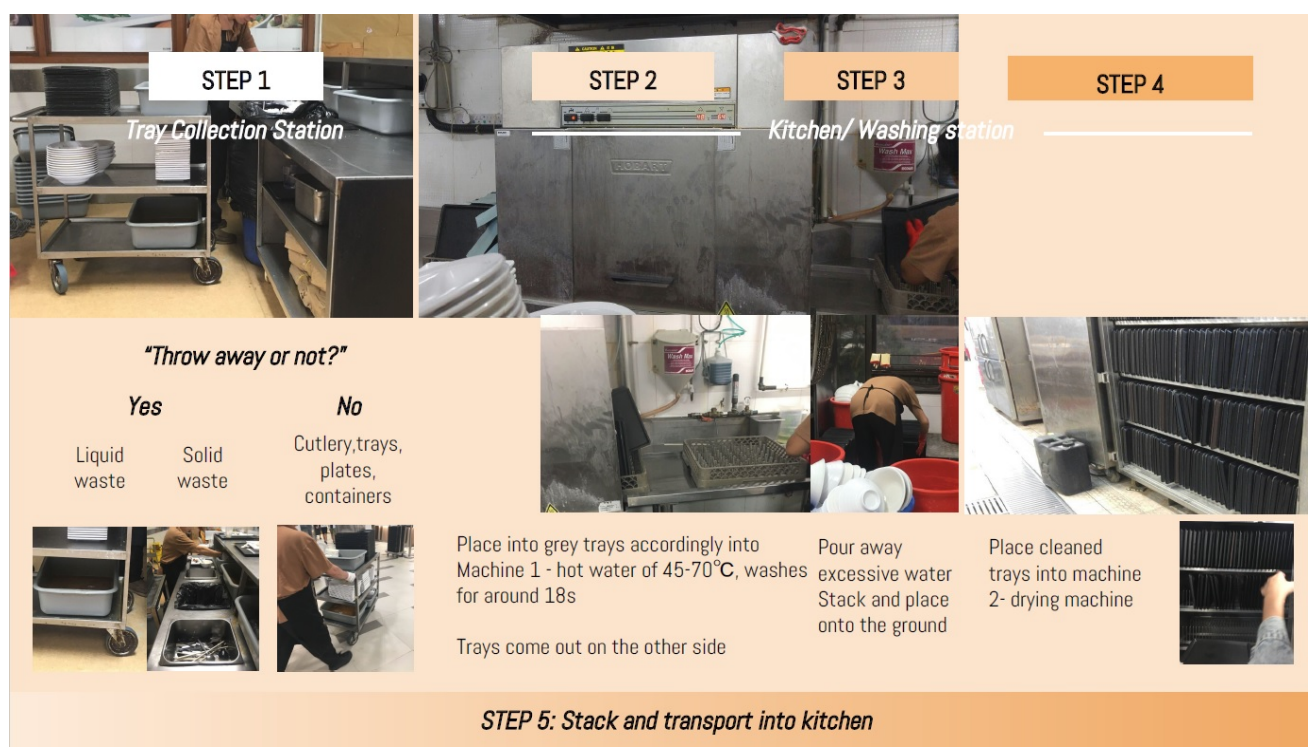

Fig. 1. Cleaning and storage process.

Overall, the research for the design criteria found that the users report issues related to balance, drink and food spillage, drink and food slippage, cumbersome and uncomfortable grip area, and a lack of space for plates and personal belongings. The grip posture and location were identified along with the body posture while carrying the food tray and setting the food tray on tables. Based on the analysis of all of the results of the methods, four main criteria for the design were identified. First, the stability of the tray and dishes set on the tray must be ensured with a slip-resistant design. Second, the design needs to allow for a flexible arrangement of food and other belongings on the tray. Third, the tray must have a hygienic surface for the safe placing of both food and belongings, including objects such as tableware and wallets. Finally, the new design must facilitate convenient after-use management and have a simple structure. After use management includes tray collection, cleaning stages, and storage. Due to the costs associated with the replacement of cleaning machinery, the design was restricted to a size which these machines could facilitate $(44.5 \mathrm{~cm}$ x $34 \mathrm{~cm})$. Additionally, a plastic based material was chosen for the design due to the manufacturing process, longevity of the product, and hygiene.

\subsection{Initial Prototype Testing}

Following development of the design criteria, three prototypes were created with a main focus on the different grip area types and a secondary focus on the eating experience (see Figure 2). Prototype 1 used a common food tray and attached handles which aimed 
to correct the flexed angles of the wrist and place them in a more neutral position whilst keeping a larger food storage space. Prototype 2 was inspired by painting palettes for the grip area and included a cutout for drink placement so as to remove the likelihood of spillage. This design also included flat edges for comfort while eating. Finally, prototype 3 removed some of the space for food to create recessed butterfly-shaped grip area while removing some of the edges. The butterfly grip area was created to support the grip while correcting awkward wrist posture and the flattened edges were created to improve the eating experience.

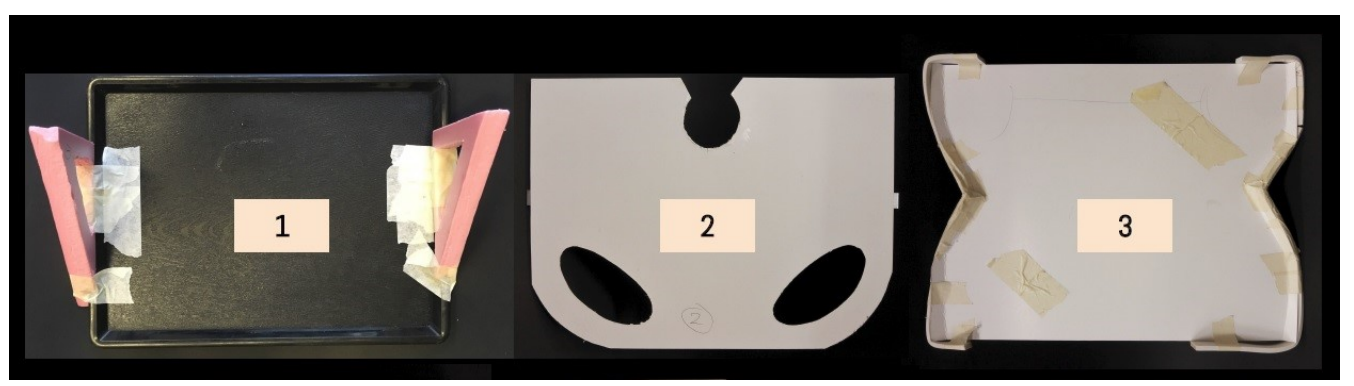

Fig. 2. Initial three prototypes.

After the prototypes were created, the three prototypes were user tested with seven participants to identify which grip area type and surface area were considered best. User testing sessions included users placing bowls with weights mimicking the weight of food on the trays, walking across a room holding the tray, placing the tray on the a table, and mimicking the eating process. Users were observed throughout the process and after were questioned on their experiences related to the grips, balance, space, and mock eating experience.

\subsection{Prototype Iteration and Testing}

Based on the findings from the initial prototype testing, prototype 3 was chosen to be expanded upon as the butterfly-shaped grip area provided a neutral position for the wrist and caused users to hold the tray closer to their body and in a more ideal position. Additionally, the butterfly-shape afforded comfort for both smaller hands and larger hands. Moreover, the space lost with the new grip design was considered acceptable to users. Finally, anti-slip materials and raised spaces for cutlery were added based on the original design criteria. 


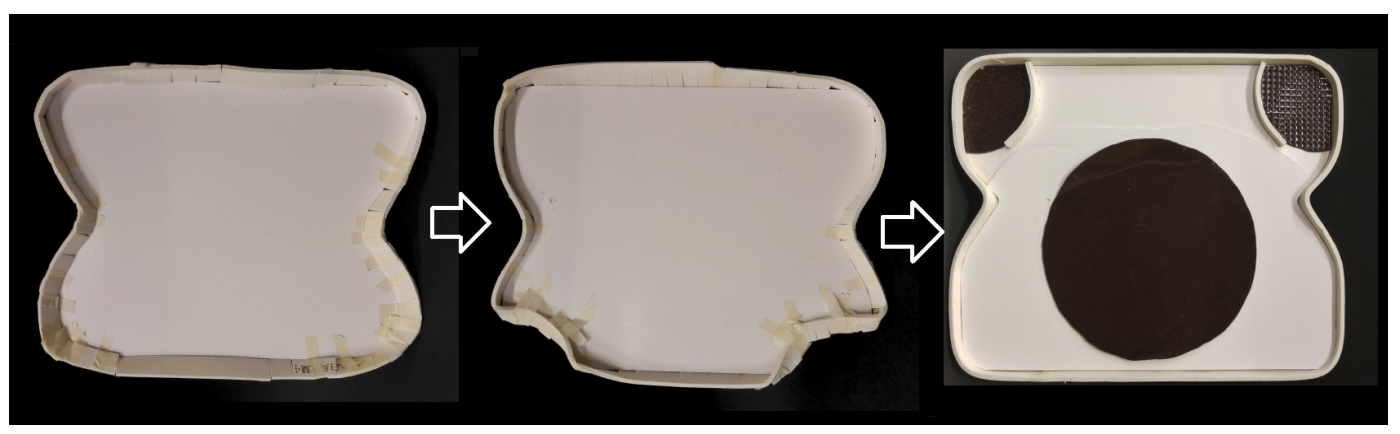

Fig. 3. Initial rapid iterations of prototypes used for testing.

After the initial grip design was decided upon, rapid prototype testing and iteration was conducted (see Figure 3). After testing, the prototypes were iterated to include a designated space for drinks, elevated cutlery rests to ensure the hygiene of the cutlery, and anti-slip material to assist in keeping the food and drink from spilling. Based on the rapid user testing, the grip area was developed further, and the bottom corners were recessed to avoid pain while eating but without lowering to the point where dishes may fall off the tray. Additionally, notches to hold the cutlery were added and a slip-resistant surface to hold dishes firmly was applied. Following the rapid prototyping, a 3D printed prototype was assembled for a final user testing session (see Figure 4). This prototype was covered fully with slip-resistant silicone.

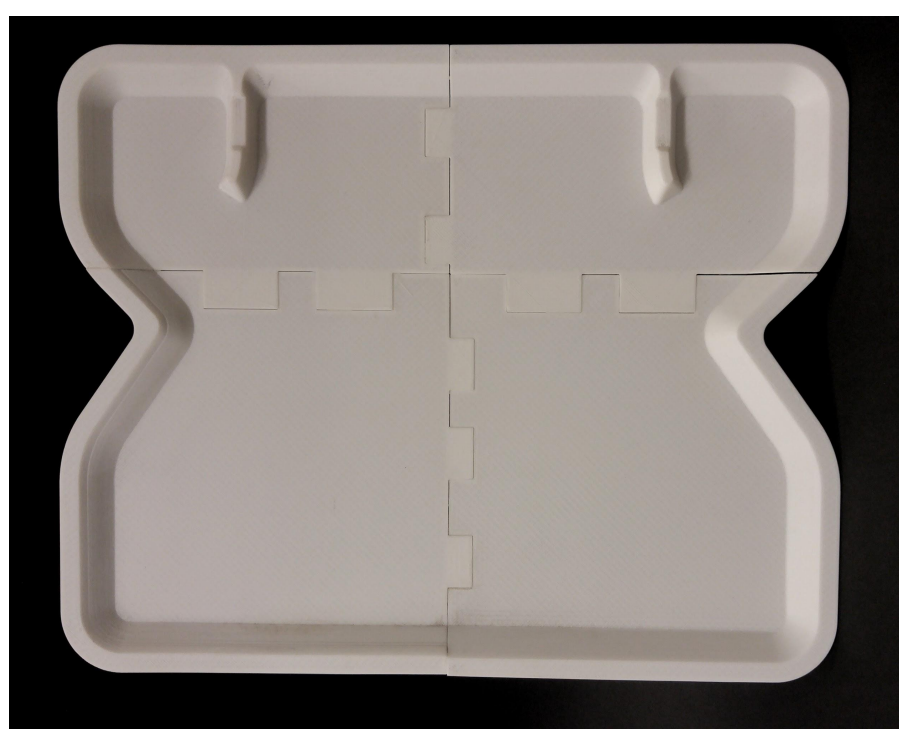

Fig. 4. 3D printed final prototype without the slip-resistant material applied.

After the final user evaluation session with three participants, the thickness of the bezel was reduced, the recessed handles were smoothed, longer and steeper notches for 
cutlery were developed, the recessed handles were moved more towards the middle for better balance, and the handles were lengthened slightly for the comfort of larger hands.

\section{$3 \quad$ Final Redesign}

The final design is slip-resistant, has improved handles, and an elevated slot for cutlery (see Figure 5). The slip-resistant silicone layer prevents cups, plates, and bowls from sliding on the tray and spilling their contents. Improved handling by a butterfly-shape recessed grip area allows users to hold the tray closer to their bodies and to the center of gravity of the tray for better balancing and a more neutral wrist position. The slot for cutlery has notches on both sides to allow users to place their cutlery on top of the elevated slots so that parts of the cutlery that are in contact with the food are raised so that they are not in contact with the tray surface. The trays remain the same width, length, and thickness so that current machinery will remain useful. The design is also stackable for easy storage.

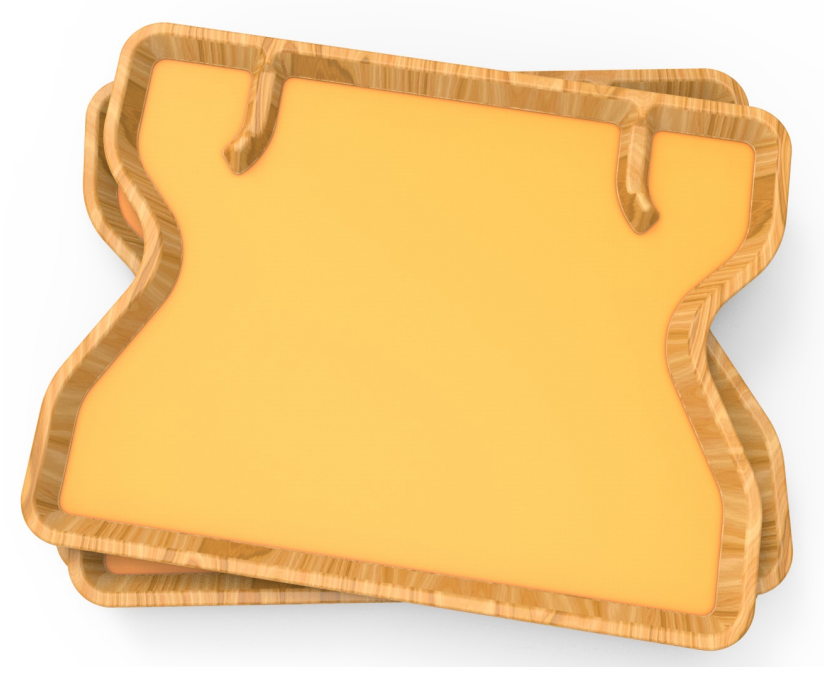

Fig. 5. Final design of the food tray.

\section{Conclusion}

Overall, a new tray was developed using ergonomics principles to ease some of the frustrations of dealing with a cafeteria style food tray. The tray was designed with the users in mind from the very first step of loading the tray with food to the cleaning process conducted by the canteen staff. The unique butterfly-shape handle was designed for better balance and more comfort when carrying the tray to a table. The material of Polypropylene for the base was chosen to be light weight and durable while a silicone layer was added to be slip-resistant. Additionally, attention was paid to the size, stack- 
ablity, and structure. This was done to facilitate convenient after-use management, storage, and to allow the trays to be used with current cleaning machinery. The ergonomically optimized design presented in this paper is an alternative to the common food tray found around the world.

Acknowledgments. The researchers would like to thank the students and canteen staff of The Hong Kong Polytechnic University for their assistance.

\section{References}

1. Gal-Oz, A., Weisberg, O., Keren-Capelovitch, T., Uziel, Y., Slyper, R., Weiss, P. L. T., Zuckerman, O.: ExciteTray: Developing an assistive technology to promote selffeeding among young children. In :Proceedings of the 2014 conference on Interaction design and children, pp. 297-300. ACM, (2014)

2. Estes, A. C.: How a Better Food Tray Is Saving Virgin Atlantic Millions, https://gizmodo.com/how-a-better-food-tray-is-saving-virgin-atlantic-millio-1588604293 Gizmodo, (2014)

3. Lee, G.: Pay a deposit for a tray? Then I won't take one, say patrons at 2 food centres, http://www.straitstimes.com/singapore/pay-a-deposit-for-a-tray-then-i-wont-take-one-saypatrons-at-two-food-centres Singapore Times, (2018)

4. Ming, T.E.: $\quad$ Pay for your tray: Two hawker centres to start charging deposits for food trays, https://www.todayonline.com/singapore/pay-your-tray-two-hawker-centres-startcharging-deposits-food-trays Times Online, (2018)

5. Bauman, C. E., Bauman, B. M.,: Food and Beverage Tray: U.S. Patent No. 4,744,597. U.S. Patent and Trademark Office, (1988)

6. Trivison, J. A.: Food Serving Tray or the Like: U.S. Patent Application No. 06/537,872. U.S. Patent and Trademark Office, (1986)

7. Wilcox, C., \& Preusser, D.: Food Tray: U.S. Patent Application No. 29/238,379. U.S. Patent and Trademark Office, (2006) 